\title{
Screening of Cellulose-degrading Fungi in Forest Litter and Fungal Effects on Litter Decomposition
}

\author{
Siqi Sun, Yuetai Weng, Xueying Di, Zhihua Liu and Guang Yang* \\ Fungi were isolated using a rose bengal chloramphenicol agar as the \\ culture medium. Congo red staining was used on sodium carboxymethyl \\ cellulose medium to screen fungal strains that have potential to produce \\ cellulolytic enzymes according to the cellulolytic index $(\mathrm{Cl})$. The ability of \\ these isolates to break down holocellulose in three forest litter substrates \\ (broad-leafed: Juglans mandshurica; coniferous: Larix gmelinii; broadleaf- \\ conifer mixed: J. mandshurica and L. gmelinii) was tested over 80 days of \\ incubation. The holocellulose content and the decomposition rule were \\ studied. The strain with the most efficient degradation effect on natural \\ cellulose in forest litter was selected. The growth of fungi was observed by \\ scanning electron microscopy (SEM). The hydrolytic circles indicated the \\ activity of cellulase produced by the fungi, and it implied that the fungi \\ could degrade cellulose. The results showed that eight strains were able \\ to degrade cellulose. The strain A2 (Peniophora incarnate) showed the \\ highest $\mathrm{Cl}$, while A4 (Sarocladium strictum) was most capable of \\ degrading holocellulose in various litter substrates. The SEM micrographs \\ revealed that A4 had the ability to invade leaf tissue and degrade \\ holocellulose in leaves. This study could be helpful for forest litter \\ management, which provides a new way to cleanup forest litter using \\ cellulose-degrading fungi.
}

Keywords: Cellulose; Decomposition; Fungi; Forest litter; Plantations

Contact information: School of Forestry, Northeast Forestry University, Harbin 150040 China;

*Corresponding author: lx_yg@163.com

\section{INTRODUCTION}

The natural decomposition of forest litter is usually a slow process, which leads to the accumulation of materials in the forest and increases the risk of forest fires when the weather is hot and dry (Nakayama and Siegert 2001). Cellulose is one of the main components of forest litter, and it largely controls the decomposition process of litter (Steffen et al. 2007; Yue et al. 2016). The litter decomposition in temperate forests is mainly affected by microorganisms including fungi, bacteria, etc. (Watling and Harper 1998; Hattenschwiler et al. 2005). Fungi degrade macromolecular compounds, such as cellulose, through enzymes secreted by fungi; in contrast, other microorganisms cannot decompose such compounds into simpler molecules. Therefore, fungi could accelerate the decay of litter (Kjøller and Struwe 1982; Djarwanto and Tachibana 2009).

The cellulose component in forest litter is not easy to break down. Therefore, screening for efficient cellulose-degrading fungi to decompose cellulose can provide a way to reduce litter accumulation, and in turn decrease forest fire risk. Highly active strains, which can completely decompose cellulose-containing substances in forest litter, are scarce, and the strains with high cellulase activity are not necessarily capable of 
decomposing forest litter (Kwon et al. 1996). Therefore, it is essential to select strains that can effectively degrade natural cellulose in litter.

Previous studies have attempted to select such active strains. Djarwanto and Tachibana (2009) collected fruiting bodies and wood material with mycelia of fungi from mangium forest plantations in south Sumatra (Indonesia). Six fungi were isolated and tested for activity to degrade mangium wood meal. Those in the genus Polyporus were the most effective fungal strain for degrading mangium lignin and holocellulose. Boberg et al. (2011) tested the ability of nine fungi isolated from coniferous litter to decompose Pinus sylvestris needles. Clavulina sistotrema strains, Chalara longipes, and three other strains within the genus Helotiales appeared to exhibit cellulolytic activity. Lophodermium pinastri (Rhytismatales) readily decomposed cellulose and caused considerable loss of lignin as well.

Given the few studies in this area, the authors screened and selected fungi from both broad-leafed and coniferous forest litters to find additional fungal species that can efficiently degrade cellulose in a variety of litter types and have potential for forest fire prevention.

\section{EXPERIMENTAL}

\section{Materials and Methods \\ Collection of fungal isolates}

The fungal isolation materials comprised the litters in the undecomposed layer $(\mathrm{L}$ layer, Litter), semi-decomposed layer (F layer, Fermentative layer), and decomposed layer (H layer, Humus horizon) (Cooke and Rayner 1985). The litters were collected from various plantations of Larix gmelinii, Juglans mandshurica, Fraxinus mandshurica, and Picea asperata Mast. in the Maoer mountain experimental forest farm of Northeast Forestry University (Harbin, China).

\section{Isolation of fungi}

Fungi were isolated using a dilute solution of rose bengal chloramphenicol agar as the culture medium (see media, below). First, impurities in the litter samples were removed, and the samples of different tree species and layers were placed in flasks containing $90 \mathrm{~mL}$ of sterile water. The flasks were incubated on a rotary shaker at $200 \mathrm{rpm}$ for $20 \mathrm{~min}$. The mother liquor was diluted to 10,20, and 30 times to form a series of concentration gradients, and approximately $200 \mu \mathrm{L}$ was absorbed and spread on the rose bengal chloramphenicol agar medium in Petri dishes and then incubated at $28{ }^{\circ} \mathrm{C}$. When the mycelium had grown on the medium, the sample was transferred to fresh potato dextrose agar medium in Petri dishes. The process was repeated until pure cultures were obtained; the purified cultures were then transferred to potato dextrose agar slants and stored at $4{ }^{\circ} \mathrm{C}$ for future use.

\section{Media}

The rose bengal chloramphenicol agar medium contained peptone $5.0 \mathrm{~g} / \mathrm{L}$, glucose $10.0 \mathrm{~g} / \mathrm{L}$, monopotassium phosphate $1.0 \mathrm{~g} / \mathrm{L}$, magnesium sulfate $0.5 \mathrm{~g} / \mathrm{L}$, agar $20.0 \mathrm{~g} / \mathrm{L}$, rose bengal $0.033 \mathrm{~g} / \mathrm{L}$, and chloramphenicol $0.1 \mathrm{~g} / \mathrm{L}$. The sodium carboxymethyl cellulose (CMC-Na) agar medium contained sodium carboxymethyl cellulose $10.0 \mathrm{~g} / \mathrm{L}$, ammonium sulfate $4.0 \mathrm{~g} / \mathrm{L}$, monopotassium phosphate $2.0 \mathrm{~g} / \mathrm{L}$, magnesium sulfate heptahydrate 0.5 
$\mathrm{g} / \mathrm{L}$, peptone $1.0 \mathrm{~g} / \mathrm{L}$, and agar $15.0 \mathrm{~g} / \mathrm{L}$. The potato dextrose agar (PDA) medium contained peeled potato $200.0 \mathrm{~g} / \mathrm{L}$, dextrose $20.0 \mathrm{~g} / \mathrm{L}$, and agar $18.0 \mathrm{~g} / \mathrm{L}$. The malt extract medium contained approximately $20.0 \mathrm{~g} / \mathrm{L}$ malt extract. All media were sterilized at $121{ }^{\circ} \mathrm{C}$ for 20 $\mathrm{min}$. All reagents were purchased from Solarbio Science \& Technology Co., Ltd. (Beijing, China).

\section{Selection of cellulose-decomposing fungi}

Cellulose-decomposing fungi were screened for activity on the CMC-Na medium using Congo red stain (AR) (Fu et al. 2010). First, pure isolates were inoculated in a triangle shape onto $\mathrm{CMC}-\mathrm{Na}$ agar medium. Then, the medium was incubated at a temperature of $28^{\circ} \mathrm{C}$. When visible colonies formed in the medium, the hydrolyzed zones were examined by saturating the CMC-Na medium with $0.2 \%$ Congo red solution for 15 min and de-staining with $0.5 \% \mathrm{NaCl}$ solution for $15 \mathrm{~min}$. The diameter of the hydrolytic circle $\left(D_{\mathrm{hc}}\right)$ around each colony was measured, and the cellulolytic index $(C I)$ of each isolate was obtained by Eq. 1,

$$
C I=\frac{D_{\mathrm{hc}}}{D_{\mathrm{fc}}}
$$

where $C I$ is the cellulolytic index, $D_{\mathrm{hc}}$ is the diameter of hydrolytic circle $(\mathrm{mm})$, and $D_{\mathrm{fc}}$ is the diameter of fungal colony $(\mathrm{mm})$.

\section{Identification of cellulose-decomposing fungi}

The fungi were identified by observing their colony characteristics and microscopic morphology such as mycelia, phialides, conidiophores, and conidia. In addition, the fungi were assessed by amplifying the ribosomal DNA (rDNA), internal transcribed spacer (ITS), and querying the GenBank database of the National Center for Biotechnology Information of the US National Institutes of Health (Bethesda, MD, USA).

\section{Degradation of litter sample}

The petioles of broad-leaves were removed, and the litter was cut into $1 \mathrm{~cm} \times 1 \mathrm{~cm}$ fragments; the coniferous litter was cut into fragments of 2-cm length. The litter samples were dried at $65{ }^{\circ} \mathrm{C}$ to a constant weight. To prepare the decomposition substrates, approximately $0.8 \mathrm{~g}$ of each coniferous and deciduous litter was placed into a $50-\mathrm{mL}$ flask that contained $10 \mathrm{~mL}$ malt extract liquid medium. Then, $0.4 \mathrm{~g}$ coniferous litter and $0.4 \mathrm{~g}$ deciduous litter were mixed, and the mixture placed into a flask to obtain a mixed decomposition substrate.

The Petri dishes of pure culture-dominant strains were opened on a clean bench, and 20 agar disks with a diameter of $6 \mathrm{~mm}$ were made in the areas where mycelia grew vigorously. The agar disks were placed in a flask containing $100 \mathrm{~mL}$ sterile water and then incubated at $28{ }^{\circ} \mathrm{C}$ on a rotary shaker at $160 \mathrm{rpm}$ for 7 days to generate suspension. The suspension was then centrifuged at $4000 \mathrm{rpm}$ for $10 \mathrm{~min}$.

The suspensions of different dominant strains were inoculated into flasks ( $300 \mu \mathrm{L}$ each) with the three decomposition substrates and cultured in a constant temperature incubator at $25{ }^{\circ} \mathrm{C}$ ( $80 \%$ relative humidity, no light). Three hundred $\mu \mathrm{L}$ of sterilized water was added into flasks containing three kinds of decomposition substrates as control treatment, and other culture conditions were the same. The degradation lasted for 80 days. Samples were taken every 20 days from three flasks for each substrate. 


\section{Scanning electron microscopy (SEM)}

The SEM analysis was used to observe the growth of fungi at various degradation stages of litter. First, the litter leaves cultured with suspensions of dominant strains were randomly selected; then, the selected leaves were cut with dissecting blades to expose the cross and vertical sections. Finally the leaves were adhered to the sample plate of the microscope. The samples were sprayed with gold using an ion sputtering instrument (JEC3000FC; JEOL Ltd., Akishima, Japan), and the sections of the leaves were observed by SEM (JSM-7500F; JEOL Ltd., Akishima, Japan).

\section{Determination of holocellulose (cellulose and hemicellulose) and lignin contents}

The holocellulose and lignin contents were determined using the fiber tester (F800; Hanon Instruments Co., Ltd., Jinan, China) (Van Soest 1963). The litter substrates were carefully removed with tweezers and placed in sterile Petri dishes. The samples were then rinsed with sterile water several times and separated using tweezers from mycelium. Initially, the treated litter samples were oven-dried to a constant weight at $65^{\circ} \mathrm{C}$. Then they were crushed into a fine powder by mortar and pestle and sieved through 40-mesh screen. The control group was treated in the same way. One gram of sample was weighed precisely and transferred to a crucible $(40 \mu \mathrm{m}$ to $100 \mu \mathrm{m})$ with ashed diatomite. The sample was digested in a digestion tube for 60 min with neutral detergent (3\% sodium dodecyl sulfate solution). After digestion, the residue, consisting of hemicellulose, cellulose, lignin, and silicate was dried and weighed. Acidic cationic detergent (2\% cetyltrimethylammonium bromide solution in $1 \mathrm{M}$ sulfuric acid) was used to digest the residue from the last step, and the residue of this digestion was composed of cellulose, lignin, and silicate. The residue from the last step was further digested with $12 \mathrm{~mol} / \mathrm{L}$ sulfuric acid at room temperature; the residue after this step contained lignin and silicate and was dried and weighed. The residue was later ashed in a muffle furnace at $550{ }^{\circ} \mathrm{C}$ for $2 \mathrm{~h}$. After cooling to room temperature, the residue was weighed, which provided the weight of silicate. The holocellulose and lignin contents were calculated using Eqs. 2 and 3, respectively,

$$
\begin{aligned}
& \text { Holocellulose content }=\frac{A-B}{m} \times 100 \% \\
& \text { Lignin content }=\frac{B-C}{m} \times 100 \%
\end{aligned}
$$

where $A$ is the mass (g) of dried hemicellulose, cellulose, lignin, and silicate, $B$ is the mass (g) of dried lignin and silicate, $C$ is the mass ( $\mathrm{g}$ ) of dried silicate, and $m$ is the sample mass, which was $1.0 \mathrm{~g}$.

The degraded holocellulose and lignin contents (relative to the control) were calculated using Eqs. 4 and 5, respectively,

$$
\begin{aligned}
& \text { Degraded holocellulose content }\left(\text { relative to the control) }=\frac{H_{c}-H_{e}}{H_{c}} \times 100 \%\right. \\
& \text { Degraded lignin content (relative to the control) }=\frac{L_{c}-L_{e}}{L_{c}} \times 100 \%
\end{aligned}
$$

where $H_{c}$ and $L_{c}$ are the holocellulose and lignin content (\%) of the control group, $H_{e}$ and $L_{e}$ are the holocellulose and lignin content (\%) of the experimental group. 


\section{RESULTS AND DISCUSSION}

\section{Screening and Isolation of Fungi}

Using the rose bengal chloramphenicol medium, 15 fungal species were isolated by colony morphology. Among these 15 isolates, four species were from the plantations of $P$. asperata Mast., six from the plantations of $F$. mandshurica, two from the plantations of $L$. gmelinii, and three from the plantations of $J$. mandshurica. The 15 species were designated as A1, A2, A3, and A4 (for P. asperata Mast.), B1, B2, B3, B4, B5, and B6 (for $F$. mandshurica), $\mathrm{C} 1$ and $\mathrm{C} 2$ (for L. gmelinii), and D1, D2, and D3 (for J. mandshurica).

\section{Selection and Identification of Cellulose-decomposing Fungi}

To test whether the isolated fungi could degrade cellulose, all 15 isolates were cultivated in CMC-Na medium. After dyeing and decolorizing, the size of the hydrolytic circle around the colony varied with the inoculated fungal species. The hydrolytic circles indicated the activity of cellulase produced by the fungi, and it implied that the fungi could degrade cellulose. The hydrolytic circles of eight isolates (A2, A3, A4, B2, B4, B6, D2, and D3), were relatively distinct, with diameters that ranged from $5.24 \mathrm{~mm}$ to $13.43 \mathrm{~mm}$ (Table 1). The $C I$ of the eight isolates ranged from 1.89 to 3.19. The B2 isolate showed the highest $C I$ value, indicating that it had the highest potential to produce cellulolytic enzymes. These isolates were identified to genus or species (Table 1) types. The eight isolates were selected for further screening.

Table 1. Fungal Colony Diameter $\left(D_{\mathrm{fc}}\right)$, Hydrolytic Circle Diameter $\left(D_{\mathrm{hc}}\right)$ on CMC$\mathrm{Na}$ Medium and Cellulolytic Index $(C l)$ for Different Fungal Taxa

\begin{tabular}{|c|c|c|c|c|}
\hline Isolates & Fungal Taxa & $D_{\mathrm{fc}}(\mathrm{mm} \pm \mathrm{SD})$ & $D_{\mathrm{hc}}(\mathrm{mm} \pm \mathrm{SD})$ & $C I(\mathrm{~mm} \pm \mathrm{SD})$ \\
\hline B2 & Cladosporium ramotenellum & $5.24 \pm 0.30$ & $16.69 \pm 0.42$ & $3.19 \pm 0.26$ \\
\hline A4 & $\begin{array}{c}\text { Sarocladium strictum (syn. } \\
\text { Acremonium strictum) }\end{array}$ & $6.48 \pm 1.06$ & $18.06 \pm 6.02$ & $2.75 \pm 0.48$ \\
\hline A3 & Pleosporales sp. & $6.66 \pm 0.63$ & $17.39 \pm 0.36$ & $2.63 \pm 0.30$ \\
\hline A2 & Peniophora incarnate & $7.84 \pm 1.71$ & $19.17 \pm 2.09$ & $2.50 \pm 0.34$ \\
\hline D2 & Fungal sp. & $13.37 \pm 0.22$ & $32.09 \pm 0.73$ & $2.40 \pm 0.04$ \\
\hline B4 & Aspergillus foetidus & $5.72 \pm 0.60$ & $12.73 \pm 0.92$ & $2.23 \pm 0.09$ \\
\hline C2 & Dothideomycetes & $11.68 \pm 0.51$ & $25.74 \pm 0.88$ & $2.21 \pm 0.06$ \\
\hline D3 & Penicillium griseofulvum & $7.58 \pm 0.30$ & $14.34 \pm 0.98$ & $1.89 \pm 0.10$ \\
\hline
\end{tabular}

SD: standard deviation

\section{Ability of Selected Fungi to Break Down Holocellulose and Lignin}

The holocellulose content of broad-leaved substrate was initially $30.4 \%$. After 20 days of incubation, the content had decreased to $28.0 \%$ to $29.5 \%$, depending on the selected fungi (Table 2), resulting in a $0 \%$ to $4.6 \%$ reduction relative to a control. Furthermore, after 80 days of incubation, the holocellulose content was $18.2 \%$ to $22.4 \%$. As shown in Table 2 , isolate A4 was the most capable of degrading holocellulose $(25.7 \%$ relative to the control), followed by A2 (24.4\% relative to the control). Isolate A3 was the least capable (8.6\% relative to the control). However, as mentioned previously, the isolate B2 showed the highest $C I$ (Table 2), indicating that the strain with the strongest cellulase activity may not have the strongest ability to decompose forest litter.

The initial holocellulose content of coniferous and mixed substrate was $37.0 \%$ and $33.4 \%$, respectively. After 20 days of incubation, the content decreased to $25.9 \%$ to $29.5 \%$ and $26.1 \%$ to $29.0 \%$, respectively (Table 2). During this period, the isolates A3 and A2 
were the most powerful at decomposing holocellulose in the two litters, respectively. Meanwhile, isolates D3 and B4 were the weakest; after 80 days of incubation, the holocellulose content was $21.8 \%$ to $26.3 \%$ and $19.8 \%$ to $26.0 \%$, respectively. The isolate A4 appeared to be the most capable of degrading holocellulose in both substrates $(30.1 \%$ and $27.2 \%$ relative to the control) as well as in the deciduous substrate. For the degradation of holocellulose, basidiomycetes were the most effective decomposers. Many of these fungi grow on cellulose-rich dead wood or leaf litter, and they use a set of hydrolytic enzymes typically consisting of endoglucanase, cellobiohydrolase, and $\beta$-glucosidase (Baldrian and Vendula 2008). From the results obtained in this study, the fungal isolate A4, selected from $P$. asperata Mast. plantation forests, was suitable for the degradation of forest litter. This is consistent with the findings of Marcelo and Renato (2016) that Sarocladium strictum, previously known as Acremonium strictum (Summerbell et al. 2011), grows relatively well on most plant-based substrates and its extracellular enzymes can break down many of these compounds before their utilization as carbon and energy sources. They also considered that the species might also be contributing to the degradation of large plant polymers. Among of the lignocellulosic biomasses studied, the sugarcane bagasse pretreated mildly was the best inductor that made the cellulase produced by $S$. strictum show the highest hydrolytic activities (Goldbeck et al. 2013). Additionally, $S$. strictum was found likely well adapted to saline environment (Cortes-Tolalpa et al. 2018), as it was previously isolated from a marine ecosystem (Fuentes et al. 2015).

Table 2. Holocellulose Content of Three Decomposition Substrates Treated with Selected Fungi

\begin{tabular}{|c|c|c|c|c|c|c|}
\hline & \multicolumn{6}{|c|}{ Holocellulose (\% \pm SD); Substrate } \\
\hline & \multicolumn{3}{|c|}{ After 20 Days } & \multicolumn{3}{c|}{ After 80 Days } \\
\hline Fungi & Deciduous & Coniferous & Mixed & Deciduous & Coniferous & Mixed \\
\hline Control & $29.4 \pm 0.1$ & $35.7 \pm 0.1$ & $32.2 \pm 0.1$ & $24.5 \pm 0.1$ & $31.2 \pm 0.1$ & $27.2 \pm 0.1$ \\
\hline B2 & $29.2 \pm 2.4$ & $29.0 \pm 7.5$ & $28.7 \pm 3.1$ & $18.8 \pm 1.7$ & $26.3 \pm 1.0$ & $22.4 \pm 6.7$ \\
\hline A4 & $28.0 \pm 0.4$ & $29.0 \pm 4.3$ & $28.0 \pm 3.2$ & $18.2 \pm 0.1$ & $21.8 \pm 1.0$ & $19.8 \pm 1.8$ \\
\hline A3 & $29.5 \pm 6.0$ & $25.9 \pm 1.1$ & $27.7 \pm 0.6$ & $22.4 \pm 0.4$ & $22.3 \pm 3.2$ & $21.7 \pm 1.5$ \\
\hline A2 & $28.1 \pm 4.4$ & $28.2 \pm 3.0$ & $26.1 \pm 0.2$ & $18.5 \pm 0.3$ & $21.8 \pm 0.1$ & $20.1 \pm 1.3$ \\
\hline D2 & $28.1 \pm 5.0$ & $28.6 \pm 2.9$ & $27.1 \pm 4.1$ & $20.5 \pm 0.8$ & $22.0 \pm 6.0$ & $20.5 \pm 3.0$ \\
\hline B4 & $28.0 \pm 3.8$ & $29.4 \pm 3.4$ & $29.0 \pm 0.9$ & $22.0 \pm 2.7$ & $23.5 \pm 2.6$ & $26.0 \pm 0.5$ \\
\hline C2 & $28.1 \pm 2.3$ & $28.8 \pm 2.1$ & $26.3 \pm 0.8$ & $20.6 \pm 2.9$ & $23.3 \pm 5.1$ & $22.9 \pm 0.9$ \\
\hline D3 & $28.5 \pm 1.3$ & $29.5 \pm 4.7$ & $28.5 \pm 4.1$ & $20.6 \pm 0.3$ & $24.7 \pm 5.0$ & $22.4 \pm 5.7$ \\
\hline
\end{tabular}

Lignin content was also measured after incubation of the eight fungi for 80 days (Table 3). This showed a different trend from the perspective of degrading holocellulose content. The lignin content of deciduous substrate was initially $42.7 \%$. After 20 days of incubation, this content increased to the range $43.3 \%$ to $52.7 \%$, depending on the fungi (Table 3). Furthermore, after 80 days of incubation, the lignin content reached $45.8 \%$ to $53.0 \%$. Similarly, the lignin content of coniferous and mixed substrate also increased during the entire experimental period. After 20 days of incubation, the lignin content increased from $44.7 \%$ and $42.5 \%$ to $45.7 \%-55.2 \%$ and $42.7 \%-49.7 \%$, respectively. After 80 days of incubation, the content had risen to $50.5 \%-54.9 \%$ and $50.3 \%-54.5 \%$. The results supported previous studies. Kirk et al. (1976) and Reid (1979) reported that lignin degradation by wood decay fungi is dependent on an additional source of carbon. Weintraub and Schimel (2003) found that during the initial stage of decomposition, the water-soluble compounds and non-lignified carbohydrates decomposed preferentially and 
their relative concentrations decreased, while lignin decomposed slightly and its relative concentration increased.

In this study, holocellulose decomposition was distinct from lignin decomposition. Berg et al. (1984) reported that, although cellulose decomposition and lignin decomposition varied greatly during early stages, their decomposition rates became nearly the same after two years.

Table 3. Lignin Content of Three Decomposition Substrates Treated with Selected Fungi

\begin{tabular}{|c|c|c|c|c|c|c|}
\hline & \multicolumn{5}{|c|}{ Lignin (\% \pm SD); Substrate } \\
\hline & \multicolumn{3}{|c|}{ After 20 Days } & \multicolumn{3}{c|}{ After 80 Days } \\
\hline Fungi & Deciduous & Coniferous & Mixed & Deciduous & Coniferous & Mixed \\
\hline Control & $42.6 \pm 0.1$ & $44.7 \pm 0.1$ & $42.4 \pm 0.1$ & $41.0 \pm 0.1$ & $43.1 \pm 0.1$ & $40.8 \pm 0.1$ \\
\hline B2 & $43.8 \pm 1.1$ & $47.1 \pm 3.0$ & $46.1 \pm 2.2$ & $45.7 \pm 8.3$ & $52.1 \pm 1.5$ & $50.3 \pm 1.0$ \\
\hline A4 & $45.2 \pm 2.7$ & $47.5 \pm 1.7$ & $45.9 \pm 0.9$ & $53.0 \pm 0.8$ & $51.2 \pm 2.2$ & $52.1 \pm 1.5$ \\
\hline A3 & $47.8 \pm 0.1$ & $51.0 \pm 1.7$ & $43.7 \pm 0.8$ & $49.4 \pm 0.4$ & $53.5 \pm 4.0$ & $54.5 \pm 2.6$ \\
\hline A2 & $45.7 \pm 1.8$ & $47.7 \pm 3.6$ & $49.7 \pm 0.9$ & $49.6 \pm 0.2$ & $54.9 \pm 4.4$ & $52.9 \pm 0.4$ \\
\hline D2 & $43.3 \pm 3.3$ & $55.2 \pm 4.4$ & $44.9 \pm 3.8$ & $53.0 \pm 5.2$ & $50.8 \pm 5.2$ & $51.6 \pm 5.1$ \\
\hline B4 & $52.7 \pm 2.0$ & $50.1 \pm 3.4$ & $46.4 \pm 0.9$ & $51.1 \pm 3.7$ & $50.5 \pm 6.0$ & $50.3 \pm 0.8$ \\
\hline C2 & $47.9 \pm 4.6$ & $47.0 \pm 1.5$ & $45.4 \pm 5.7$ & $47.1 \pm 7.6$ & $54.7 \pm 5.7$ & $50.8 \pm 0.2$ \\
\hline D3 & $47.3 \pm 5.3$ & $45.7 \pm 9.9$ & $42.7 \pm 1.5$ & $52.5 \pm 6.1$ & $50.9 \pm 2.2$ & $50.6 \pm 1.8$ \\
\hline
\end{tabular}

\section{Observation by SEM}

This study found that the fungal strain A4 surpassed the other strains and exhibited stronger ability to break down holocellulose.

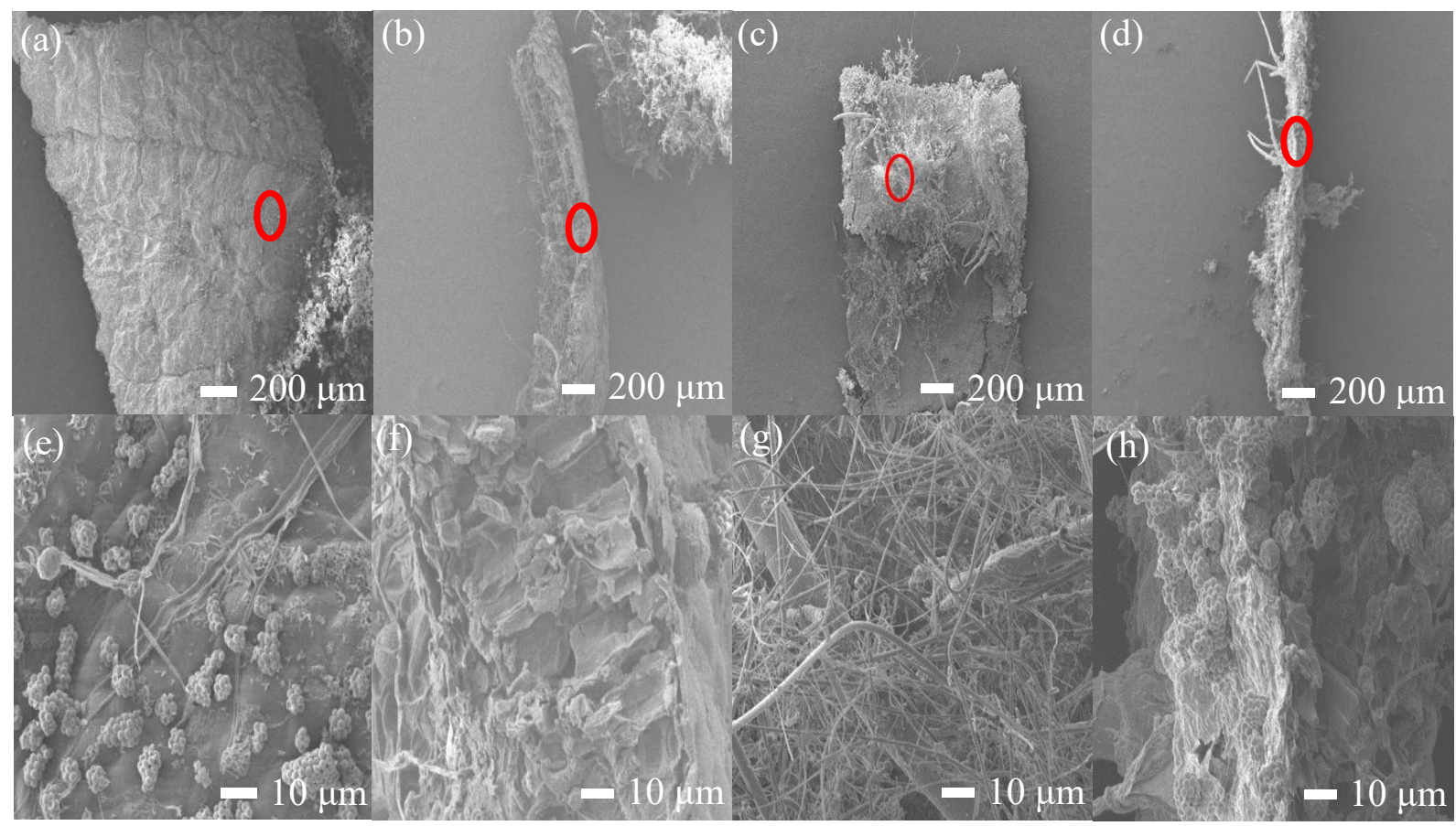

Fig. 1. Scanning electron micrographs of Juglans mandshurica leaf sections after $20 \mathrm{~d}$ and $80 \mathrm{~d}$ of incubation of Sarocladium strictum (isolate A4): (a) Leaf surface after 20 days of incubation; (b) Leaf longitudinal section after 20 days of incubation; (c) Leaf surface after 80 days of incubation; (d) Leaf longitudinal section after 80 days of incubation; (e), (f), (g), and (h) show details from the magnified red circles in (a), (b), (c), and (d), respectively 
The SEM analysis revealed the growth of its hyphae, adhering to the leaf surface and invading leaf tissues. As shown in Fig. 1(a), after 20 days of incubation, there were fewer mycelia on the leaf surface, and the mycelial morphology was simple. Slender phialides arose from aerial hyphae and could be observed in Fig. 1(e). Ellipsoidal conidia appeared as grouped in slimy heads (Giraldo et al. 2015) and scattered on the leaf surface after being released from the phialides, resembling strings of grapes. However, as shown in Figs. 1(b) and 1(f), no obvious hyphae and conidia were observed in the longitudinal section of the blade. As shown in Fig. 1(c), after 80 days of incubation, the mycelia covered the entire leaf blade and formed a dense network. The finger-shaped branches of the hyphae extended along the leaf surface. The mycelia were developed, and they became thicker, with a large number of conidia attached, as shown in Fig. 1(g). In the longitudinal section of the blade, a small amount of mycelia began to invade the tissues, and a few attached conidia were found in Fig. 1(h). The micrographs revealed that strain A4 was a powerful holocellulose-degrading fungus, which had the ability to invade leaf tissue and degrade holocellulose in leaves. This confirmed the initial finding that fungus A4 was most capable of degrading holocellulose.

\section{CONCLUSIONS}

1. The results of this study demonstrated that Sarocladium strictum (A4), was the most effective agent to degrade the natural cellulose in forest litter in the study area.

2. Among the 15 selected fungi, eight were able to degrade cellulose: Cladosporium ramotenellum (B2), Sarocladium strictum (A4), Pleosporales sp. (A3), Peniophora incarnate (A2), Fungal sp. (D2), Aspergillus foetidus (B4), Dothideomycetes (C2), and Penicillium griseofulvum (D3).

3. Although Peniophora incarnata (A2) showed the highest cellulolytic index, its ability to degrade cellulose in litter was weaker than Sarocladium strictum (A4), meaning that the isolate with the strongest cellulase activity may not have the strongest ability to decompose forest litter.

4. The scanning electron micrographs indicated that the mycelia of Sarocladium strictum (A4) adhered to the leaf surface and invaded the leaf tissue, degrading holocellulose by secreting cellulolytic enzymes.

\section{ACKNOWLEDGMENTS}

This research was supported by the National Key R\&D Program of China during the $13^{\text {th }}$ Five-Year Period [2017YFD0600106-2] and the National Nature Science Foundation of China [31870644]. 


\section{REFERENCES CITED}

Baldrian, P., and Vendula, V. (2008). "Degradation of cellulose by basidiomycetous fungi,” FEMS Microbiology Reviews 32(3), 501-521. DOI: 10.1111/j.15746976.2008.00106.x

Berg, B., Ekbohm, G., and McCalugherty, C. (1984). "Lignin and holocellu!ose relations during long-term decomposition of some forest litters. Long-term decomposition in a Scots pine forest. IV". Canadian Journal of Botany 62(12), 2540-2550. DOI: 10.1139/b84-345

Boberg, J. B., Ihrmark, K., and Lindahl, B. D. (2011). "Decomposing capacity of fungi commonly detected in Pinus sylvestris needle litter," Fungal Ecology 4(1), 110-114. DOI: $10.1016 /$ j.funeco.2010.09.002

Cooke, R. C., and Rayner, A. D. M. (1985). "Ecology of saprotrophic fungi," Journal of Applied Ecology 22(2), 604-605. DOI: 10.2307/

Cortes-Tolalpa, L., Norder, J., van Elsas, J. D., and Falcao Salles, J. (2018). "Halotolerant microbial consortia able to degrade highly recalcitrant plant biomass substrate," Applied Microbiology and Biotechnology 102(6): 2913-2927. DOI: 10.1007/s00253017-8714-6

Djarwanto, and Tachibana, S. (2009). "Screening of fungi capable of degrading lignocellulose from plantation forests," Pakistan Journal of Biological Sciences 12(9), 669-675. DOI: 10.3923/pjbs.2009.669.675

Fu, X., Liu, P., Lin, L., Hong, Y., Huang, X., Meng, X., and Liu, Z. (2010). “A novel endoglucanase (Ce19P) from a marine bacterium Paenibacillus sp. BME-14," Applied Biochemistry and Biotechnology 160(6), 1627-1636. DOI: 10.1007/s12010-0098648-2

Fuentes, M. E., Quiñones, R. A., Gutiérrez, M. H., and Pantoja, S. (2015). "Effects of temperature and glucose concentration on the growth and respiration of fungal species isolated from a highly productive coastal upwelling ecosystem," Fungal Ecology 13, 135-149. DOI: dx.doi.org/10.1016/j.funeco.2014.09.006

Giraldo, A., Gené, J., Sutton, D. A., Madrid, H., de Hoog, G. S., Cano, J., Decock, C., Crous, P. W., and Guarro, J. (2015). "Phylogeny of Sarocladium (Hypocreales)," Persoonia 34(1), 10-24. DOI: 10.3767/003158515X685364

Goldbeck, R., Ramos, M. M., Pereira, G. A. G., and Maugeri-Filho, F. (2013). "Cellulase production from a new strain Acremonium strictum isolated from the brazilian biome using different substrates," Bioresource Technology 128, 797-803. DOI: 10.1016/j.biortech.2012.10.034

Hättenschwiler, S., Tiunov, A. V., and Scheu, S. (2005). "Biodiversity and litter decomposition in terrestrial ecosystems," Annual Review of Ecology, Evolution and Systematics 36, 191-218. DOI: 10.1146/annurev.ecolsys.36.112904.151932

Kirk, T. K., Connors, W. J., and Zeikus, J. G. (1976). "Requirement for a growth substrate during lignin decomposition by two wood-rotting fungi," Applied Environmental Microbiology 32(1), 192-194. DOI: 10.1128/AEM.32.1.192-194.1976

Kjøller, A., and Struwe, S. (1982). "Microfungi in ecosystems: Fungal occurrence and activity in litter and soil," Oikos 39(3), 389-422. DOI: 10.2307/3544690

Kwon, G. S., Moon, S. H., Hong, S. D., Lee, H. M., Kim, H. S., Oh, H. M., and Yoon, B. D. (1996). "A novel flocculant biopolymer produced by Pestalotiopsis sp. KCTC8637,” Biotechnology Letters 18, 459-1464. DOI: 10.1007/BF00129355 
Marcelo, E. F., and Renato, A. Q. (2016). "Carbon utilization profile of the filamentous fungal species Fusarium fujikuroi, Penicillium decumbens, and Sarocladium strictum isolated from marine coastal environments," Mycologia, 108(6), 1069-1081. DOI: $10.3852 / 15-338$

Nakayama, M., and Siegert, F. (2001). "Comparative study on C and L band SAR for fire scar monitoring," in: The 22nd Asian Conference on Remote Sensing, Singapore, pp. 516-519.

Reid, I. D. (1979). "The influence of nutrient balance on lignin degradation by the whiterot fungus Phanerochaete chrysosporium," Canadian Journal of Botany-Revue Canadienne De Botanique 57(19), 2050-2058. DOI: 10.1139/b79-255

Steffen, K. T., Cajthaml, T., Šnajdr, J., and Baldrian P. (2007). "Differential degradation of oak (Quercus petraea) leaf litter by litter-decomposing basidiomycetes," Research in Microbiology 158(5), 447-455. DOI: 10.1016/j.resmic.2007.04.002

Summerbell, R. C., Gueidan, C., Schroers, H.-J., de Hoog, G. S., Starink, M., Rosete, Y. A., Guarro, J., and Scott, J. A. (2011). "Acremonium phylogenetic overview and revision of Gliomastix, Sarocladium, and Trichothecium," Studies in Mycology 68:139-162. DOI: 10.3114/sim.2011.68.06

Van Soest, P. J. (1963). "Use of detergents in the analysis of fibrous feeds. II. A rapid method for the determination of fiber and lignin," Journal of the Association of Official Analytical Chemists 46(5), 829-835.

Watling, R., and Harper, D. B. (1998). "Chloromethane production by wood-rotting fungi and an estimate of the global flux to the atmosphere," Mycology Research 102(7), 769-787. DOI: $10.1017 / \mathrm{s} 0953756298006157$

Weintraub, M. N., and Schimel, J. P. (2003). "Interactions between carbon and nitrogen mineralization and soil organic matter chemistry in arctic tundra soils," Ecosystems 6(2), 29-143. DOI: $10.2307 / 3658812$

Yue, K., Peng, C., Yang, W., and Peng, Y. (2016). "Degradation of lignin and cellulose during foliar litter decomposition in an alpine forest river," Ecosphere 7(10), e01523. DOI: $10.1002 /$ ecs2.1523

Article submitted: December 10, 2019; Peer review completed: February 29, 2020; Revised version received and accepted: March 8, 2020; Published: March 10, 2020. DOI: 10.15376/biores. 15.2.2937-2946 